\title{
Decision support using the Multistatic Tactical Planning Aid (MSTPA)
}

\author{
Christopher Strode • Baptiste Mourre • Michel Rixen
}

Received: 14 January 2011 / Accepted: 26 July 2011 / Published online: 30 August 2011

(C) The Author(s) 2011. This article is published with open access at Springerlink.com

\begin{abstract}
The Multistatic Tactical Planning Aid (MSTPA) is a tool currently in development at NATO Undersea Research Centre which may be used to model the performance of a given multistatic sensor network in terms of the probability of detection of a submarine, the ability to hold a track and whether such a track could be correctly classified as such. The tool therefore considers the entire chain of events from an initial calculation of signal excess, the generation of a contact considering localisation errors, followed by the subsequent tracking and classification process. In its current form, the tool may be used to plan a particular multistatic scenario through operational analysis of many Monte Carlo simulations. The future development of MSTPA will transition towards a real-time decision support tool to assist operators and planners at sea. This study introduces a number of generic decision support techniques which may be wrapped around the MSTPA tool. The acoustic performance metric that will drive decisions will of course be subject to uncertainty relating to environmental measurements and extrapolations. The effect of this uncertainty on acoustic performance is examined here. Future studies will consider the sensitivity of the eventual decision-in terms of optimum sensor positions - to the acoustic uncertainty.
\end{abstract}

Responsible Editor: John Osler

This article is part of the Topical Collection on Maritime Rapid Environmental Assessment

C. Strode $(\varangle) \cdot$ B. Mourre $\cdot$ M. Rixen

NATO Undersea Research Centre

Viale S. Bartolomeo 400, 19126 La Spezia, Italy

e-mail: strode@nurc.nato.int
Keywords Decision support - MSTPA • Game theory $\cdot$ Optimisation $\cdot$ Uncertainty

\section{Introduction}

The Multistatic Tactical Planning Aid (MSTPA) is a tool currently in development at NATO Undersea Research Centre (NURC) which may be used to model the performance of a given multistatic sensor network in terms of the probability of detection of a submarine, the ability to hold a track and whether such a track could be correctly classified. The tool considers the entire chain of events from an initial calculation of signal excess (Harrison 2002), the generation of a contact considering localisation errors (Coraluppi 2003), followed by the subsequent tracking and classification processes (Wathelet et al. 2006, 2008b). The premise behind the MSTPA model is to model each part of the chain in relatively simple terms, whilst maintaining sufficient fidelity from signal excess to eventual classification. This methodology drives MSTPA towards its goal of becoming a decision support tool as opposed to a purely acoustic model. A more detailed description of MSTPA and the algorithms it employs may be found in Wathelet et al. (2006).

The MSTPA tool is primarily designed to assess the performance of multistatic scenarios in which, by definition, sources and receivers may be spatially distributed on separate platforms. Such a disposition is known to increase area coverage and decrease the ability of a submarine to evade detection since many acoustic pathways exist between it and the receivers. However, the performance increase comes at the cost 
of increased operational complexity due to the many possible sensor geometries and the need for reliable underwater communication and coordination between the source and receivers. As nations begin to procure sonar systems with a multistatic capability, there is a very real need for a tool such as MSTPA to automate the planning process and to increase the situational awareness and understanding of its operators.

The MSTPA tool must consider the optimisation of both static and dynamic multistatic networks. The static type might include a barrier to detect the transit of a threat submarine through a given area. A dynamic scenario might consider the persistent deployment of a number of autonomous vehicles with multistatic capability in order to act as a reactive area search network. This scenario is currently receiving much attention at NURC as part of the cooperative anti-submarine warfare project in which a distributed and persistent network of autonomous sensors is envisaged (Hughes et al. 2010; Been et al. 2010). This scenario will include the use of static sources combined with a number of mobile platforms towing arrays with which to make multistatic detections. The MSTPA tool may be used as a test bed for AUV behaviours in which realistic false contacts are generated in order to test the ability of such behaviours to react to the true target whilst discriminating against false contacts and tracks. This report will consider mostly the static deployment case in order to decrease the number of variables to optimise such that the concepts of decision support may be investigated (see Section 3).

In essence, the aim is to investigate generic decision support techniques that may be 'wrapped around' the existing MSTPA model (acting as a central metric calculator) in order to aid the decision-making process. This must begin with the automated extraction and analysis of environmental data-whether measured, modelled or forecast, such that the relevant information is passed onto the MSTPA model. Already, we see that the MSTPA acoustic, tracking and classification engine becomes just one module within a greater chain of decision support techniques. This must be the case, since any optimisation or decision-making process must at some point determine the effectiveness of given options. It is here that the existing MSTPA modules must calculate relevant metrics for the particular scenario being considered. The results from the MSTPA model will be integrated with techniques to analyse the expected threat decisions, automatically re-examine the scenario and re-plan accordingly to determine a best solution. This process will feature elements of both decision theory-in order to mathematically describe the options and choices available to both threat and friendly forces (and to rate their utility) - and game theory in order to play out the potential combinations of threat and friendly options until an optimum is determined.

Recent advances within the core acoustic element of the model allow for the ingestion of fully 3-D temperature and salinity data as may be measured at sea. The recent REP10 experiment demonstrated rapid environmental assessment in which glider measurements, satellite measurements and advanced oceanographic models were combined in a super-ensemble to produce accurate 3-D maps of temperature and salinity, with forecasts up to $72 \mathrm{~h}$. Initial results are presented in which the uncertainty inherent in the oceanographic measurements and forecasts are transitioned into the final acoustic coverage product. Environmental uncertainty is represented here through the 3-D superensemble (3DSE) uncertainty and is sampled in order to generate a number of candidate temperature and salinity fields. Each is then used to generate a signal excess coverage map from which the final operational uncertainty (the detectability of a submarine) may be determined.

The final step in the decision support chain is the presentation of results to the operator. At this point, a number of human factors come into consideration when attempting to present complicated information to operational personnel. There are two broad approaches to the eventual output of a decision aid. Firstly, the tool may simply output what it has determined to be the 'answer'. This may be the optimal positions for all sources and receivers within the scenario and the operator need only implement this plan. Secondly, the tool might display the performance metrics for a number of different solutions allowing the operator to make the final decision. This second option is most likely to be preferred by the operational community who are intelligent, well-trained professionals that are used to making difficult decisions. At this point, the distinction between a decision support tool and a decision making tool becomes apparent. The tool should assist decisions by presenting the relevant information together with candidate solutions such that the operator still has the final say. A tool acting as a black box which spits out answers is unlikely to be trusted.

The following broad topics will be investigated during the course of this paper, and their application towards transitioning MSTPA into a decision support tool will be discussed:

- Game theory

- Optimisation

- Oceanographic uncertainty 


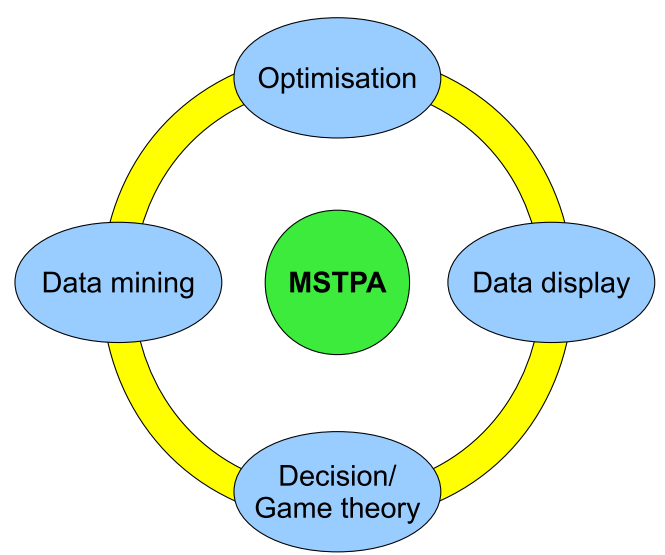

Fig. 1 Decision support framework

The above processes will be wrapped around the existing MSTPA modules as described in Fig. 1. It is hoped that the additional modules will be sufficiently generic such that they may be quickly applied to additional scenarios-such as glider path planning — simply by replacing the core metric calculating module, that is, replacing a multistatic model with a model of glider dynamics and data sampling requirements such that the same decision theory modules may be applied but with different calculations of measures of effectiveness. The ability to transform a scientific tool into decision support software is a powerful ideal that will facilitate the rapid transition of scientific research towards operational relevance.

\section{Decision support concepts}

Before examining some of the decision support techniques in more detail, let us first examine just what is meant by decision support and what exactly consti- tutes a good decision. Decision support systems are a core subject area of the information systems discipline and at the most fundamental level are concerned with representing and processing knowledge for the purpose of improving decision making. An understanding of decision support must include that of decisions and decision making which in turn requires an understanding of knowledge and knowledge management. A decision support system is a computer-based system that represents and processes knowledge in ways that allow decision making to be more productive, agile, innovative and/or reputable (Burstein and Holsapple 2008). It is therefore no surprise that the field of data mining (the first module in our proposed decision support chain) may be considered as the extraction of knowledge/information from data. Van Lohuizen identifies a progression of six states of knowledge beginning with data, through information, and culminating in a decision as shown in Fig. 2 (Wali Van Lohuizen 1986).

A decision is regarded as a choice between a number of courses of action, such as the strategies that may be employed by the decision maker. In the field of decision theory, the decision maker (or rational agent) will choose the strategy with the greatest expected utility (Von-neumann and Morgenstern 1953) —utility being the measure of satisfaction derived from each outcome. A decision support tool mimics this method in that it considers all possible choices, assessing each according to some metric (the utility) and determining the best. A computer-based system is well suited to assessing a large number of options in a systematic manner and presenting this information to the decision maker. In fact, the tool need not return a single best solution but a number of alternatives for the decision maker to compare using the system calculated metrics. This highlights the nature of the proposed tool as being a decision support tool and not necessarily a decision making tool.
Fig. 2 Knowledge as a progression of states

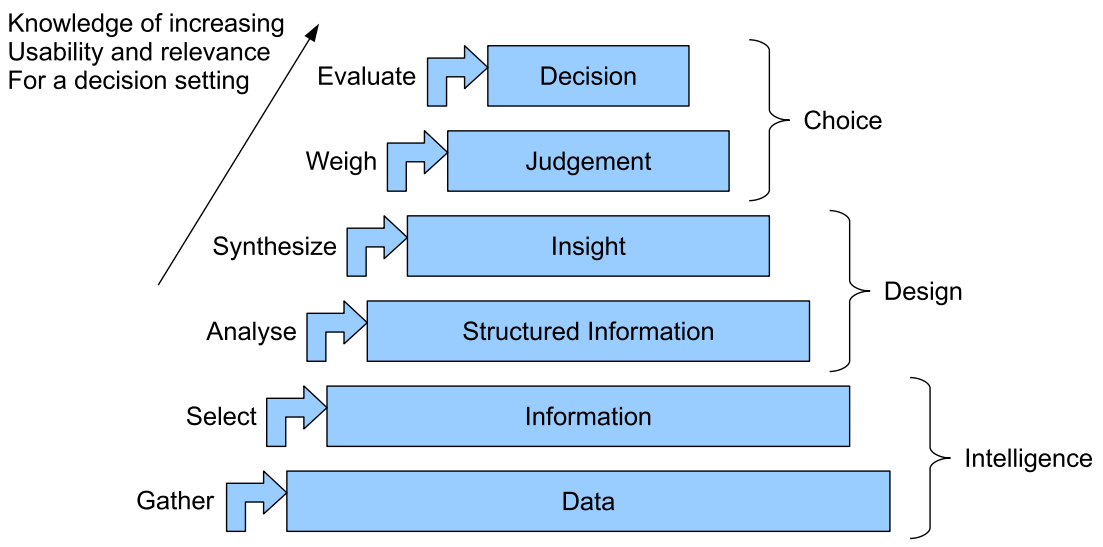


In the military context in which our scenario lies, a black box spitting out a single decision (instruction) to well-trained intelligent personnel is unlikely to be trusted and will eventually be ignored. Presenting alternative solutions along with their respective metrics (time permitting), especially those that could not be determined in the operators head, is a far more sensible approach.

The presentation of supporting evidence along with a decision may help to alleviate a common problem with decision support systems. The problem is that of the cognitive bias of the operator, whereby he may be expecting a particular solution and be unwilling to accept any alternative (Davis et al. 2005). Such a bias can arise from many factors including habit in which familiar results are given more weight based on previous outcomes. In the military scenarios considered here, the decision support tool may have to operate in the face of ingrained doctrine and training. If a result is presented which appears to contradict this, perhaps because complicated environmental factors were previously unaccounted for, the operator may question the result. As soon as a result is questioned, the trust in the tool is compromised and it may soon be ignored all together. This problem may be countered by supplementing results with evidence in the form of a metric calculation and possible alternative courses of action with lower metrics. In effect, the operator is brought into the decision process and is allowed to make up his own mind with help from the tool.

There is then a balance which must be found between the level of information presented by the tool and the level of understanding required by the operator. As already discussed, a single answer determined by the tool requiring no thought on the part of the operator is not an ideal situation-nor is displaying all outcomes with some metric that is not understood by the operator. This suggests a two-pronged approach to improving automated decision support tools:

1. The tools must be made more user-friendly.

2. The user should be trained to understand the underlying assumptions/metrics of the tool.

In effect, the goal is to optimise the fit between the decision tool and its user by developing the tool and training the operator. Current decision science recommends a number of ways to increase the usage and trustworthiness of these tools through the following design features (Davis et al. 2005):

1. Allow personalisation-decision makers rate a tool as more usable when they can customize certain features.
2. Use graphical interfaces-decision makers rate graphical tools as more trustworthy than text-based modules.

3. Maintain interactivity-decision makers prefer interactive tools to non-interactive tools.

4. Limit pre-processing of data-decision makers prefer decision support calculations (such as ranking alternatives) that can be easily linked to concepts in the 'hard data'. They should have access to all of the underlying data used by the tool.

We will leave the precise definition of our performance metric until the next section where we consider the scenario in more detail. However, regardless of the metric, we may elaborate on what is a good decisionor at least what exactly the proposed decision support system should achieve. To this end, two desirable outcomes are considered:

1. The performance metric is better than an otherwise manual solution.

2. The solution is derived faster than a manual solution.

Generally, the tool should decrease the effort required to plan a mission whilst at the same time result in better operational performance. We might expect, for example, a reduction in the level of resources required (platforms, fuel, staffing etc.) to fulfil a given mission. This leads towards two forms of optimum result: one in which the mission outcome is the same but required less resources and another in which the mission outcome is itself improved.

The second point above is an important one in that it considers the time taken to generate a decision. Military forces seek to win an engagement by making decisions quicker than the enemy using a concept known as the OODA loop, standing for observe, orient, decide and act (developed by military strategist and USAF Colonel John Boyd). Friendly forces seek to 'get inside' the enemies OODA loop by making their decisions faster to gain the advantage (Rosenberg 2010). In fact, making a less optimum decision faster than the enemies optimum decisions can allow for friendly forces to out manoeuvre the enemy and gain the upper hand. This particular way of thinking applies mostly to more dynamic scenarios and threats than will be considered in the context of this study. However, since we seek to produce generic modules that may be applied to any scenario, the speed at which decisions are made should be considered. One possible scenario that is not far removed from that considered here is the optimum deployment and behaviour of a fleet of autonomous underwater vehicles. If the decision aid can react to 
changes in the environment and platform positions faster than the threat submarine, then the system have the advantage.

An important note should be made regarding the problem of uncertainty within decision support systems, in particular, the effect of environmental uncertainty on sonar performance-crucial for the scenarios considered here. For example, the translation of uncertainty in sound velocity profile (as determined by interpolation from glider tracks) to uncertainty in signal excess is examined here. However, this relates to a single step within the decision support process, and further studies will examine the flow of uncertainty from input data to eventual optimum decision. The problem of uncertainty in both input data and output decisions might require a search for a strategy that is most robust. In this case, an optimum solution is one that is least susceptible to the inevitable uncertainties within the decision process. As an example, consider the problem of mine clearance. A mine hunter might plan a mission and, on completion, report that the area clearance is between $65 \%$ and $70 \%$. This accurate result will allow the planners of the operation to conduct further mine hunting operations and allow for the eventual passage of follow-on traffic through the area. However, a clearance result of between $50 \%$ and $90 \%$-although on average higher-reduces the confidence of the mission planners and does not allow for accurate follow on planning.

\section{The scenario}

The baseline scenario considered in this study is that of the deployment of a fixed network of multistatic sonar sensors attempting to detect the transit of a submarine through an area of interest. This scenario type is referred to as a barrier and is common in anti-submarine warfare (ASW) operations whereby a submarine is suspected to transit through a well-defined area. Constricted transit through a specified area may occur due to geographical narrowing of the water space-a choke point, or perhaps at the exit of a port or harbour facility. Such a scenario serves to decrease a number of variables and results in a more manageable scenario with which to begin the transition of MSTPA towards decision support software.

A barrier scenario allows for a well-defined scenario area through which a target must transit. This leads to a better understanding of target behaviour/mission since it must in general enter one side of the scenario area and exit at the opposite end. This is in contrast to an area search problem in which no such assumption may be made as to the general motion of the target which might even chose to remain stationary in order to remain undetected for as long as possible.

A further assumption is that the receiving nodes are covert-the submarine cannot detect them. This is an important assumption and one that is often quoted as being an inherent advantage of multistatic systemsthe inability of a target to determine where the receivers are located will limit his ability to counter them and thus raise the performance of the network. In such a scenario, the target will of course be aware of the presence of the sound source, by counter detecting its transmission, but will have no way of determining any information about the number (if any) and position of the distributed network of multistatic receivers.

Having settled upon a particular scenario (problem), a suitable performance metric must be defined. We consider the number of detections, track initiations or successful classifications of a submarine for a given multistatic sensor configuration. Such a metric also leads to the corresponding measure of success for the threat, this being the negative of the network success metric. These metrics lead nicely into the domain of game theory as will be seen in Section 4 .

\section{Game theory}

Game theory is an extension of decision theory in which the utility (measure of success) of a player's strategy is a function of the strategy chosen by the other player. The sensor placement scenario is a two-player game in which one-the target-seeks to minimise the number of times he is detected-whilst the other player-the network planner-seeks to maximise the number of detections. Since the metrics of the two players are completely opposed, the game is non-cooperative. In such a game, each player must have at least an eye on minimising the payoff to the other player whilst still seeking to maximise his own. The following discussion presents initial analysis combining the use of the $\mathrm{A}^{*}$ path planning algorithm within a game theoretic setting (Strode 2009).

The A* algorithm may be described as follows: Given a weighted directional graph $G$ with a distinguished start node $s$ and a set of goal nodes $\tau$, the optimal path problem is to find a least-cost path from $s$ to any member of $\tau$, where the cost of the path may, in general, be an arbitrary function of the weights assigned to the nodes and branches along that path (Dechter $1985)$. The algorithm applies an additive evaluation function $f(n)=g(n)+h(n)$, where $g(n)$ is the cost of 
the currently evaluated path from $s$ to $n$ and $h(n)$ is a heuristic estimate of the cost of the path remaining between $n$ and any member of $\tau$.

Let us assume that both the multistatic network planner and submarine commander are aware of the existence of a barrier consisting of a source and three receivers-though not their actual location. This is a necessary assumption of equal knowledge and is essential to solving the game. We begin the analysis by considering a total of eight possible sensor geometries using an ad hoc design-the process may be applied to any number of geometries within the constraints of processing time (the current analysis is completed in $7 \mathrm{~min}$ ). Note that in some cases a receiver is placed at the same position as the source resulting in a combined monostatic and multistatic network. The submarine is assumed to enter the scenario area, detect and localise the source and thereafter choose a strategy - or pathwith which to transit the barrier. The submarine uses the $\mathrm{A}^{*}$ path planning algorithm to determine his optimal track against each possible sensor geometry (G1G8) using the technique described in Strode (2009). The resulting optimum tracks-those with the least detections-are shown in Fig. 3 for all eight potential network geometries.

Of course, the submarine commander cannot know which geometry has been deployed, nor can the multistatic network planner know which track the submarine will adopt. Consequently, both players must determine the number of detections for each optimised track against all network geometries. In other words, what happens if the submarine chooses to optimise against G3 but the planner chose G6? The game theoretic element comes into play when we consider the following thought process - the target can optimise perfectly against a particular geometry but the planner knows this and so will not lay it. In effect, we consider a more realistic level of threat intelligence in which the submarine does not have complete knowledge of the network-and therefore cannot simply generate a single optimum track through it.

A game theoretic solution is only as complete as the consideration of the various strategies that might be employed by each player. For instance, might we consider a further random path or straight path which the target could employ as a strategy against the network? This is a common problem when setting up a game theoretic solution-not considering all possible choices. However, in this case, we are giving the target the ability to generate an optimum track in all cases. We know from Strode (2009) that the resulting A* paths are optimum and always better than a straight (or any other) path.
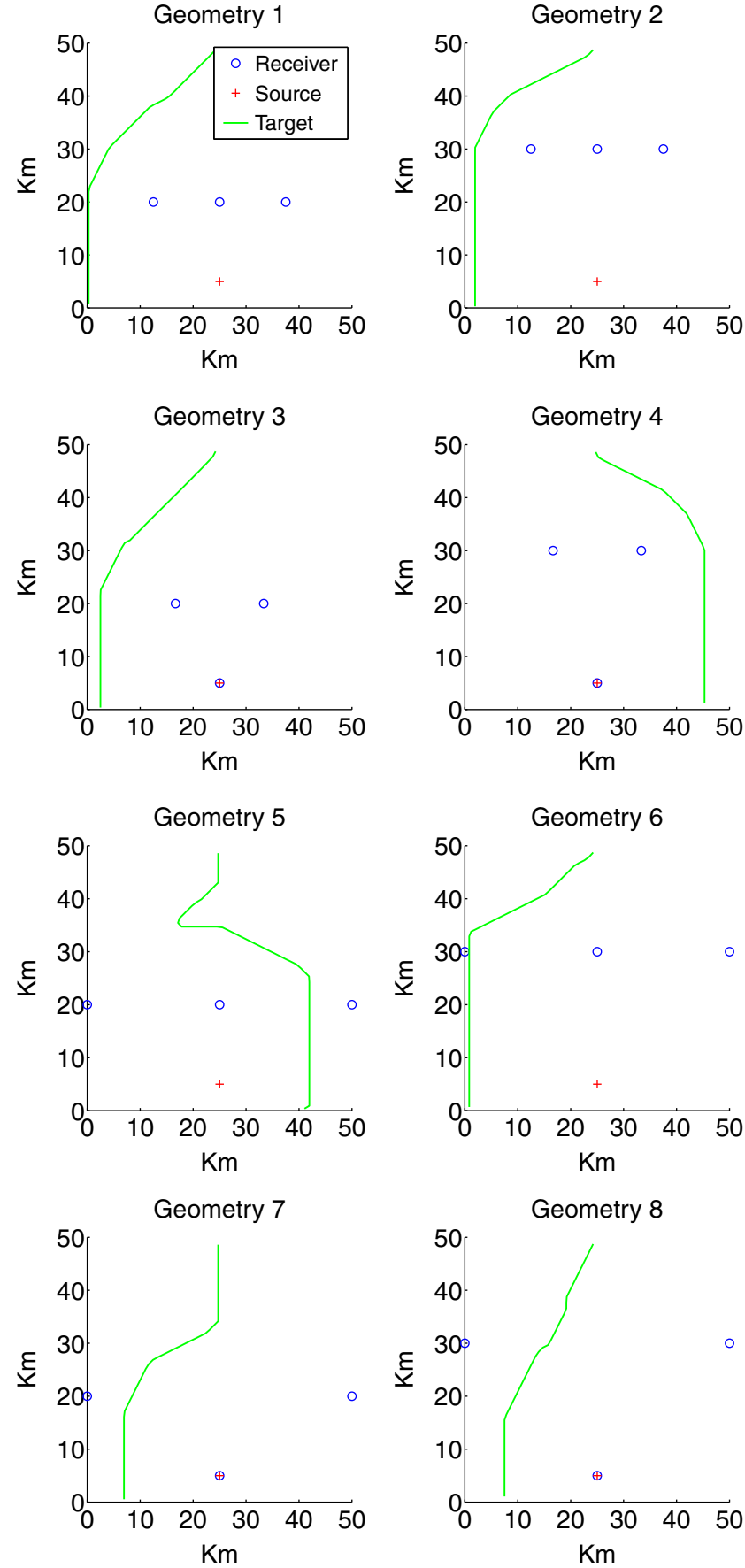

Fig. 3 Optimised path for target transiting from top to bottom through barrier

Suppose we add an additional column to the decision matrix for a straight line transit through the network. Since the $A^{*}$ track is optimal, the payoff to the target for the straight track must be worse. In fact, even if the straight track was optimal, the A* would have returned that same track. Therefore, all payoffs for the straight line tactic will be worse or the same as that of the $\mathrm{A}^{*}$ 
equivalent. The straight line tactic is then dominated by the $\mathrm{A}^{*}$ tactic and can be removed from the matrix without affecting the outcome of the game. This is the case for any other potential tactic that can be envisaged. Consequently, we need not consider any other target tactics. The only room for improvement is to consider more and more potential geometries-planner strategies. A further study will examine the robustness of the mixed strategy to additional geometries-in effect, the optimum spatial resolution of potential sensor positions must be determined. The combination of optimum path generation with a game theoretic approach is a powerful one employing intelligent path planning whilst taking into account the target's incomplete knowledge of the network geometry.

The number of target detections for all combinations of planner and target strategy are given in matrix format in Table 1. The score within each element of the array gives the payoff to the planner-the payoff to the target is the negative of the displayed value. In this way, the planer seeks to maximise the number in the array whilst, at the same time, the target seeks to minimise. This game may be described as two player, non-cooperative, zero sum. The matrix given in Table 1 is said to be the normal form of the game in which the payoff to the row player (the planner) is given. The matrix is later referred to as $a_{i j}$ with subscripts $i$ and $j$ relating to rows (planner strategy) and columns (target strategy), respectively.

What information can be derived from Table 1? The aim is of course to determine the optimal sensor geometry to deploy-in a game theoretic sense, this will be a compromise between the conflicting aims of both planner and target. A quick analysis reveals that, as expected, if the target optimises against the correct geometry (values along the diagonal), the number of detections is reduced, usually to zero. Geometries 5 and 6 would appear to be the best option from the planner's point of view since, even if the target correctly optimises against them, he is still detected. However, if a particular geometry was an obvious choice for the planner, the target would simply optimise correctly and the result of the game would be (at best) a single detection. In keeping with the premise of MSTPA, a single detection is unlikely to allow the planner to react against the threat and generate a suitable fire control solution-requiring the generation of a track and subsequent classification as a threat. Game theory may be used to solve the decision matrix in such a way as to find the optimal solution for the planner-and by extension the target.

It can also be seen that, on occasion, a target optimising against the wrong geometry still remains undetected. For example, had the target chosen to optimise against G4 but the network was in fact $\mathrm{G} 2$ he is still undetected. This analysis might suggest that geometry G2 should not be adopted by the planner since there are two possible outcomes resulting in zero target detections.

Inspection of Table 1 reveals that no strategies for either planner or target are dominated-that is always equal or worse than any other. The solution of this game is expected to be a mix of strategies for both planner and target. Since if a single geometry was the planners best strategy, the target could counter it and guarantee a worst case of a single detection. Many games of this type result in a mixed solution by which each strategy is given a probability that it should be employed. In this way, the target cannot know exactly which geometry will be deployed and must in turn implement his own mixture of strategies. This is an interesting result since it hints towards the value of the covert receivers. We know that if the target could determine the location of the receivers, he could optimise against the geometry and greatly reduce the number of detections. The game theoretic solution acknowledges this fact and therefore returns a mixture of strategies for the planner in order to maintain an 'element of surprise' over the target.

The result of the game theoretic solution, assuming it is greater than 1 , provides a convenient measure of the value of covertness. A normal form matrix, as given in
Table 1 Matrix game for a barrier scenario (showing payoff to planner)

\begin{tabular}{lrrrrrrrr}
\hline $\begin{array}{l}\text { Planner strategy } \\
\text { (geometry to lay) }\end{array}$ & \multicolumn{8}{l}{ Target strategy (geometry to optimise against) } \\
\cline { 2 - 9 } & G1 & G2 & G3 & G4 & G5 & G6 & G7 & G8 \\
\hline G1 & 0 & 14 & 0 & 10 & 16 & 14 & 16 & 9 \\
G2 & 9 & 0 & 5 & 0 & 13 & 9 & 13 & 9 \\
G3 & 11 & 21 & 0 & 15 & 15 & 16 & 15 & 9 \\
G4 & 15 & 11 & 8 & 0 & 13 & 14 & 13 & 11 \\
G5 & 19 & 29 & 22 & 42 & 1 & 20 & 9 & 11 \\
G6 & 10 & 12 & 7 & 7 & 10 & 1 & 10 & 1 \\
G7 & 23 & 20 & 14 & 19 & 10 & 16 & 0 & 0 \\
G8 & 20 & 17 & 17 & 17 & 14 & 14 & 9 & 0 \\
\hline
\end{tabular}


Table 1, may be solved using linear programming techniques (Owen 1995), whereby a mixed strategy over the set of pure strategies can be found that optimises the expected payoff to both players. A mixed strategy is a vector defining the probability with which each pure strategy should be played. If the planner uses the mixed strategy $x_{i}=\left(x_{1}, \ldots, x_{m}\right)$, he can assure himself of an expectation at least equal to $\lambda$, where $\lambda$ is any number such that $\sum a_{i j} x_{i} \geq \lambda$ for $j=1, \ldots, n$ where $a_{i j}$ is the matrix given in Table 1 , represented mathematically below.

$a_{i, j}=\left(\begin{array}{cccc}a_{1,1} & a_{1,2} & \cdots & a_{1, j} \\ a_{2,1} & a_{2,2} & \cdots & a_{2, j} \\ \vdots & \vdots & \ddots & \vdots \\ a_{i, 1} & a_{i, 2} & \cdots & a_{i, j}\end{array}\right)$

Thus, the problem of obtaining an optimal strategy for the planner reduces to the linear program

Maximise $\lambda$ subject to

$$
\begin{array}{rlrl}
-\sum_{i=1}^{m} a_{i j} x_{i}+\lambda & \leq 0 & j & =1, \ldots, n \\
\sum_{i=1}^{m} x_{i} & =1 & \\
x_{i} & \geq 0 & i & =1, \ldots, m
\end{array}
$$

Similarly, the solution for the target reduces to the linear program

Minimise $\mu$ subject to

$$
\begin{array}{rlr}
-\sum_{j=1}^{n} a_{i j} y_{j}+\mu & \geq 0 & i=1, \ldots, m \\
\sum_{j=1}^{n} y_{j} & =1 & \\
y_{i} & \geq 0 & j=1, \ldots, n
\end{array}
$$

Solving the linear program using the Matlab toolbox results in the following mixed strategy for the planner:

$x=(0.1,0.0,0.0,0.7,0.2,0.0,0.0,0.0)$

whilst the target will employ the mixed strategy

$y=(0.0,0.0,0.2,0.1,0.4,0.0,0.0,0.3)$

The value of the game derived from the above solution is 10.6, this being the average number of target detections made by the network. Note that, had the target known the position of the receivers, he would have been detected no more than once. This then measures the value of the covertness of the network.

A question arises, however, as to the meaning of this average result and indeed the probability distribution over geometries, for a network that will be laid only once. Whilst a number of threat submarines may indeed attempt to penetrate the barrier over time-applying an appropriate strategy mix - the network itself is deployed only once and is thereafter fixed. Consequently, a strategy mix for the planner might seem rather meaningless-except that it suggests he should lay one of several geometries-thereby depriving the threat of complete knowledge. The decision support tool can of course roll a dice to determine which of the geometries the planner should lay according to the mixed strategy solution. The important point is that the target cannot know which geometry was laid, even if he used the same decision support tool whilst pretending to be the planner. This concept is known as a 'chance device' (Wagner et al. 1999) and ensures that the opponent cannot predict the outcome.

Williams (1954) states:

Consider a non-repeatable game which is terribly important to you, and in which your opponent has excellent human intelligence of all kinds. Also assume that it will be murderous if your opponent knows which strategy you will adopt. Your only hope is to select a strategy by a chance device which the enemies intelligence cannot master-he may be lucky of course and anticipate your choice anyway but you have to accept some risk. Game theory simply tells you the characteristics your chance device should have.

It is possible that the game theoretic approach be applied to the more dynamic scenario in which the behaviour of mobile sensors is considered. In this case, a normal form matrix could be envisaged in which sensor strategies are no longer a fixed position but a complete description of a particular behaviour. For example, a behaviour may be a simple close range approach or the maximise signal-to-noise ratio (SNR) behaviour described in LePage (2010). Similarly, target behaviours could range from simple random course alterations (assuming he has not detected the mobile sensor) or maximise range (assuming he has detected the sensor). A normal form payoff matrix, containing all combinations of sensor and target behaviour, could be determined from simulations and solved using the technique described above. 


\section{Optimisation}

Work has been conducted on the addition of optimisation routines to the MSTPA tool. In particular, the use of genetic algorithms (GA) to optimise static sensor placement and dynamic platform waypoints has previously been reported in Wathelet et al. (2008a).

The approach taken in MSTPA to determine the optimum placement of selected ASW assets against a threat submarine is to maximise a number of measures of effectiveness (MOE). Current MOEs include the detection area coverage and the probability that a submarine will be detected or tracked before crossing a barrier. Optimisation algorithms are used in MSTPA to determine the best placement of the ASW assets by maximising these MOEs.

MSTPAs optimiser uses a set of heuristic optimisation algorithms. These are used to maximise the MOEs that describe the ASW mission of interest. The missions currently in MSTPA include static area surveillance, barrier operation and force protection. The MOEs that relate to these missions are, respectively, area cover- age, barrier coverage and area coverage around a high value unit.

MSTPA maximises the MOEs, namely the detection area coverage and the probability that a submarine will be detected or tracked before crossing a barrier, using a set of optimisation algorithms from the JavaEvA (a Java implementation of Evolutionary Algorithms) open source library (Streichert and Ulmer 2005). Although many algorithms are included as part of this library, such as simulated annealing, hill climbers and Monte Carlo search, the main technique used and reported in Wathelet et al. (2008a) is a GA.

The basic concept of a genetic algorithm is to loosely model the principle of evolution through natural selection. The algorithm represents a typical solution to the problem as a vector of source and receiver positions. A population of these individuals is created, and operators such as mutation and recombination are applied on the population of individuals to arrive at an individual that maximises the fitness function. For example, in the area coverage scenario, the GA will determine the $x$ and $y$ position of each asset that maximises the area coverage function.
Fig. 4 Sequential output from MSTPA genetic algorithm

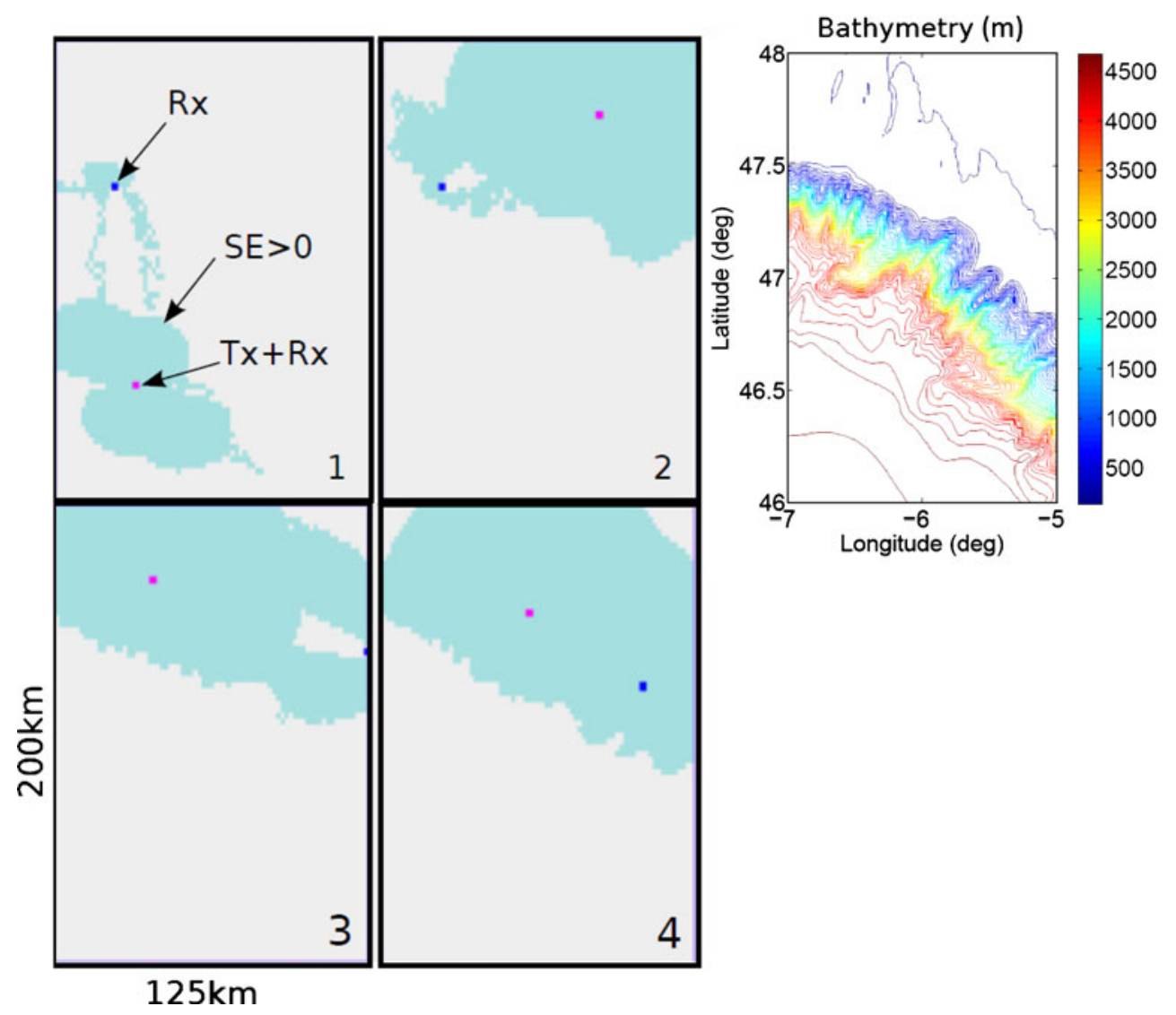


The example shown in Fig. 4 shows intermediate sensor positions generated by the genetic algorithm (labelled 1-3) on its way towards finding the optimal solution (4). The performance metric in this case is the area of the scenario with a signal excess value greater than 0 (shaded blue). The scenario includes a monostatic sensor $(\mathrm{T} x+\mathrm{Rx})$ with an additional multistatic receiver ( $\mathrm{Rx}$ ) which may be placed anywhere in the scenario area. The environment is characterised by an iso-velocity sound speed profile over a continental shelf bathymetry shown in the figure.

The GA solution shown in Fig. 4 generated 1,000 candidate geometries on its way towards the optimal solution. The area coverage metric for each geometry required $75 \times 75$ signal excess calculations. The solution therefore required over 5.5 million signal excess calculations, each taking into account range-dependent bathymetry. The iso-velocity sound speed assumption, combined with the fast closed-form algorithms described in Harrison (2002), allowed for the solution to be obtained in only $7.5 \mathrm{~min}$. Signal excess is determined to be the difference between the actual SNR and that required for the sonar system to register a detection. The acoustic propagation and reverberation is modelled using a mode stripping approach with Lambert's law scattering (Harrison 2002).

When modelling individual targets transiting through a network, there are a number of options as to what their precise behaviour may be. The simplest approach is to model a large number of targets on different (but unchanging) headings. For each geometry, the percentage that were detected may be determined and used as a cost measure within an optimisation routine. When considering a barrier scenario in which targets transit through a network from 'top' to 'bottom', perhaps on headings between $160^{\circ}$ and $200^{\circ}$, it was often the case that a large proportion of targets would have been tracked at some point during their transit. This can give a false impression of the effectiveness of the network due to the assumption that all targets are completely unaware of its presence and continue 'blindly' on. This type of threat behaviour is unlikely considering the ability to counter detect the source.

There is then a scale of threat intelligence that may be applied to the problem of optimising network performance. The simplest and least intelligent threat is the straight running one already discussed. We might then imagine targets that are aware of the transmission of the source and attempt to evade in some reactive way. Lastly, as a worst-case (most intelligent) target, we might consider one with complete knowledge of the network and an ability to generate an optimum (e.g. least detectable) path through it. A network that is optimised against such a worst-case target is likely to be the most robust.

The worst-case, most intelligent threat has previously been modelled using the $\mathrm{A} *$ algorithm with which it determines an optimal path through the network according to a number of metrics (Wathelet et al. 2008a; Strode 2009). The combination of the $A^{*}$ algorithm with the game theoretic approach detailed in Section 4
Fig. 5 Typical glider track showing temperature measurements

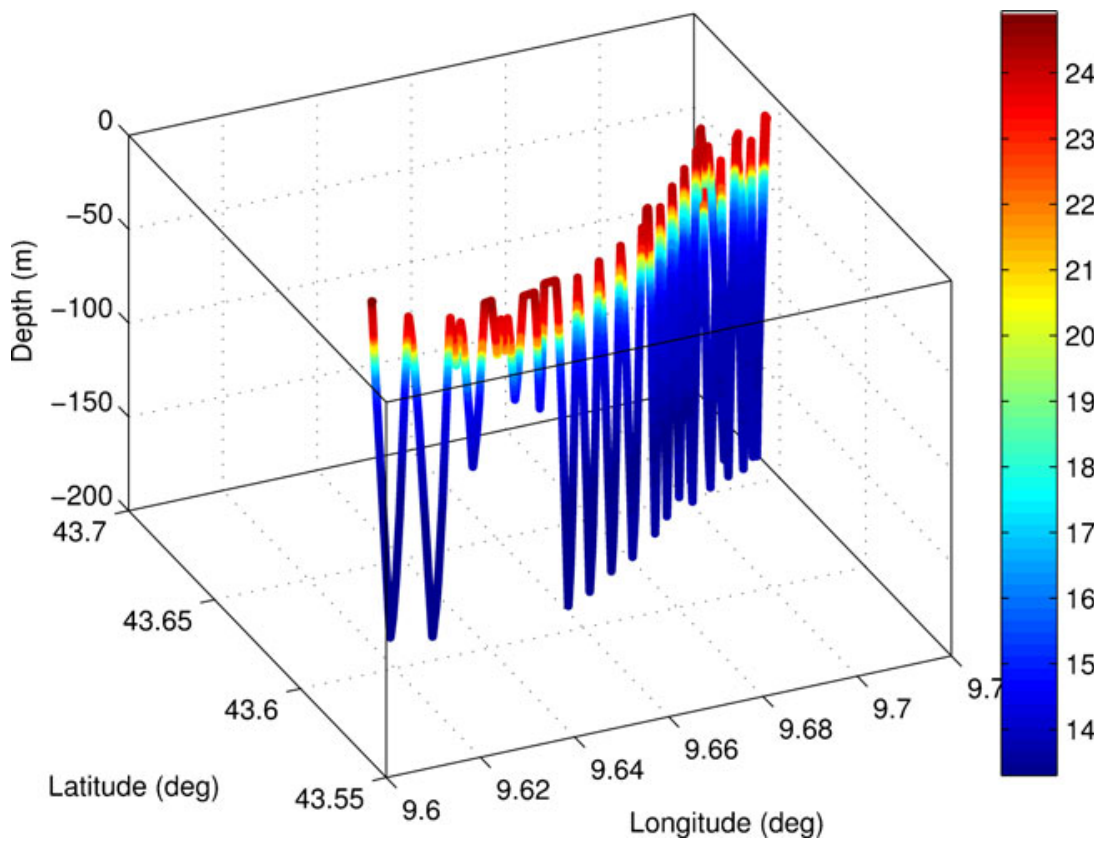


Fig. 6 3-D sound speed volume from super-ensemble output with bathymetry

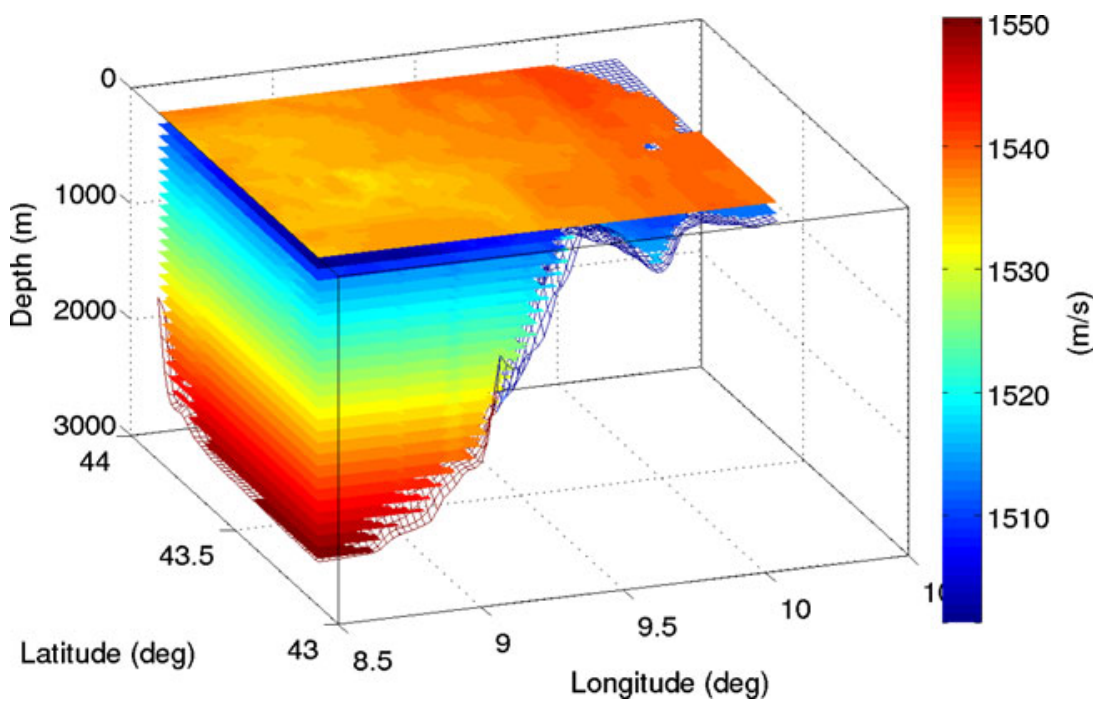

developed for the iso-velocity case and maintains a significant speed advantage over other tools. The ability to generate a fast acoustic performance map over a scenario area which includes realistic bathymetry and fully range-dependent temperature and salinity (and by extension sound speed) allows for more accurate calculations of operational performance metrics. However, the ability of the model to account for environmental information at ever-increasing resolutions leads to questions of the ability of surface platforms to adequately measure the environment.

An ASW surface platform will typically deploy an expendable bathythermograph (XBT) probe in order to determine the variation of water temperature with
Fig. 7 Ten sound speed (metres per second) profile samples at 25 locations uniformly distributed over scenario area (north to top) (sound speed profiles show top $200 \mathrm{~m}$ of water column only)
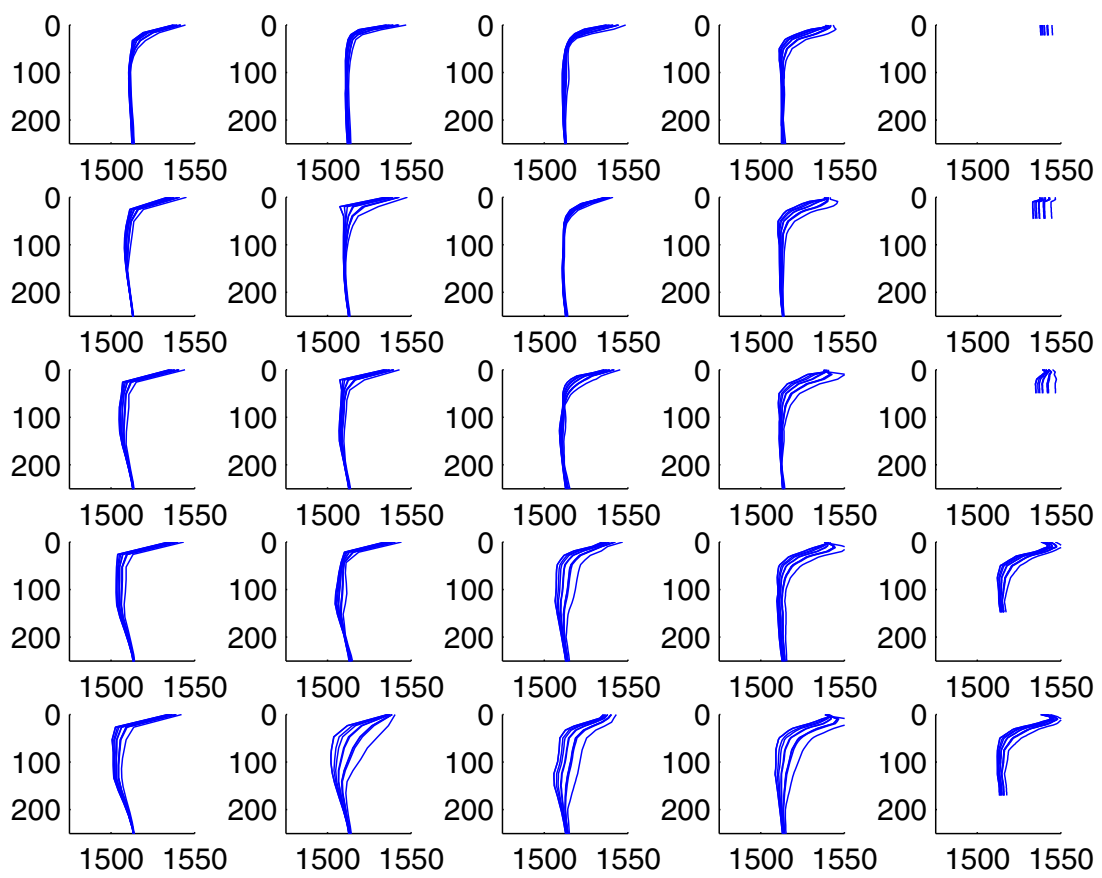
depth at a particular location within the scenario area. The temperature profile is converted to a sound speed profile and is assumed to apply to the whole scenario area which could be well in excess of $100 \mathrm{~km}^{2}$. Acoustic calculations are performed to determine the performance of a given sonar sensor against a target of a particular target strength-in which the propagation of sound is strongly affected by the gradient of the measured sound speed profile. It is common knowledge, amongst oceanographers and acousticians alike, that the undersea environment is complex and dynamic and that a sound speed profile measured at a particular point cannot be applied to the scenario area as a whole.

A tool such as MSTPA can account for as many discrete sound speed profiles as can be measured over a scenario area, perhaps by a number of distributed XBTs. Additionally, the tool can infer such profiles from 3-D measurements of temperature and salinity using the Chen-Millero formula (Chen and Millero 1977). Of course, no measurement device can cover an entire scenario area in both position and depth to obtain a true picture of the complex underwater environment. To address this shortfall, NURC is currently employing underwater gliders to make persistent measurements of temperature and salinity over large areas at various depths due to the continuous diving and surfacing movement method they employ. However, even these data do not cover all points in position and depth within a scenario area-a typical glider measurement profile is shown in Fig. 5, colour coded according to the measured temperature value. Intelligent extrapolation of the glider measurements is required to provide oceanographic information at all points within the water volume. However, any such
Fig. 8 Signal excess (decibels) samples
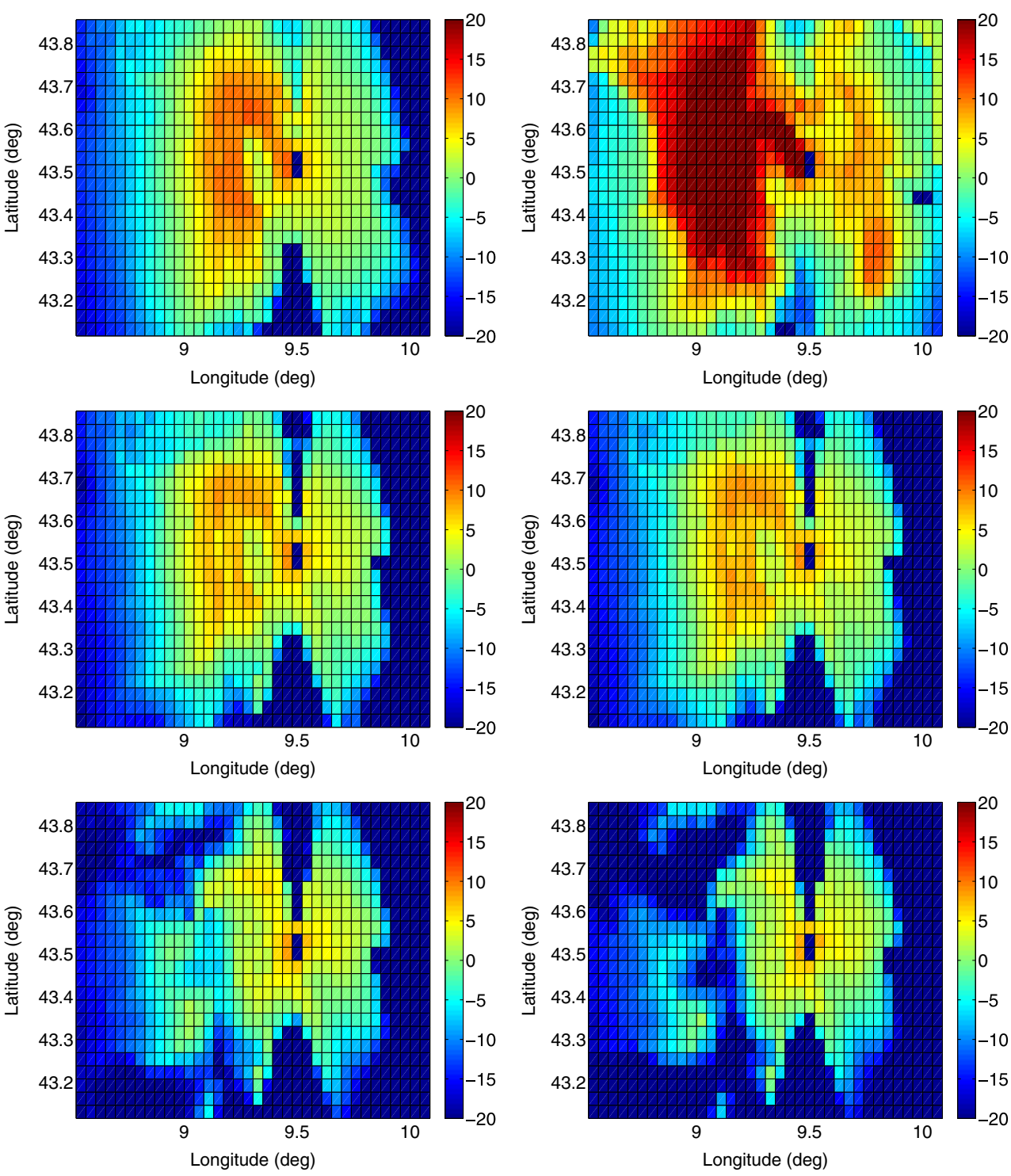
extrapolation process introduces uncertainty as the range from measured values increases.

Initial results are presented here to investigate the impact of uncertainty in sound speed-due to the necessary extrapolation of measured values of temperature and salinity-on the eventual operational performance metric. The performance metric considered here is that of the predicted signal excess in decibels for a submarine of constant $10-\mathrm{dB}$ target strength operating at a depth of $20 \mathrm{~m}$. Such an acoustic calculation is strongly dependent on variations in sound speed in both range and depth as sound is propagated from the sonar sensor.

The sound speed profiles used in this study were deduced from 3DSE temperature and salinity predictions. The 3DSE method (Lenartz et al. 2010) combines several ocean model predictions using optimal weights computed during a recent past learning period when observations are available. In the present implementation, three high-resolution $(1-\mathrm{km})$ models are used: Regional Ocean Modelling System run at NURC, 3D Model for Applications at Regional Scale provided by PREVIMER (www.previmer.fr) and Navy Coastal Ocean Model run at the Naval Research Laboratory. The 3DSE forecast is associated with an uncertainty es- timate based on the assimilation of observations during the learning period.

The approach to be followed here is that of perturbing the input temperature data according to the estimated uncertainty at each point. Each temperature perturbation is fed into MSTPA, along with the mean salinity profile, in order to determine the full 3-D sound speed profile. This results in a number of possible signal excess maps from which the mean and standard deviation may be extracted. A convenient single output - capturing both the mean and uncertainty of all perturbations-is the probability of detection. This calculation considers all perturbed signal excess values in a cell and determines the fraction with positive values. A positive value of signal excess, by definition, will result in a detection of the target submarine.

Figure 6 shows an example of the 3-D sound speed volume resulting from the super-ensemble temperature and salinity output. The data shown in Fig. 6 may be perturbed, according to the estimated uncertainty, to generate a number of probable outcomes. For this preliminary analysis, the data were sampled 100 times over 2 standard deviations - each point within the full 3 -D sound speed volume is perturbed assuming a normal distribution. Figure 7 shows the first $250 \mathrm{~m}$ of ten

Fig. 9 Signal excess statistics

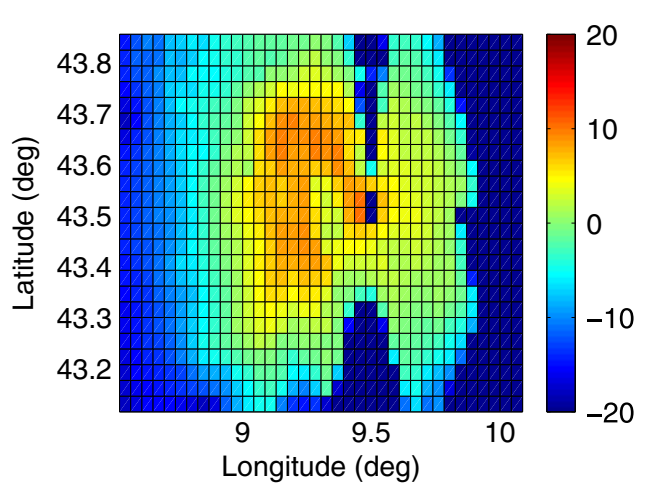

(a) Mean signal excess (dB)

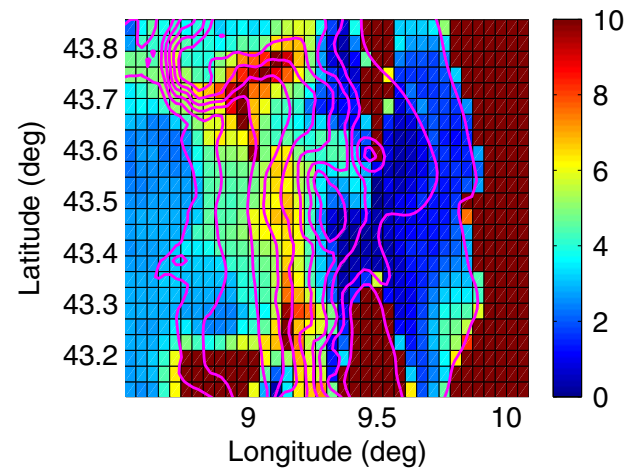

(b) Standard deviation of signal excess (dB) showing bathymetry contours

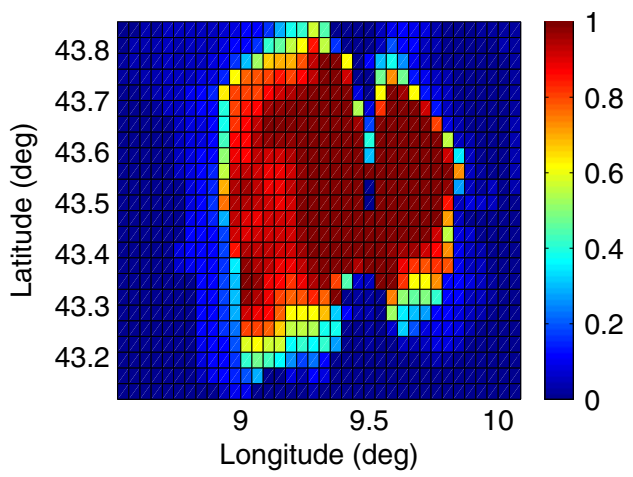

(c) Probability of detection 
perturbed sound speed profiles at 25 locations uniformly distributed around the scenario area. Note that in some locations the bathymetry is less than $250 \mathrm{~m}$ resulting in shortened profiles.

All 100 sound speed profiles were then used to generate 100 signal excess coverage maps, of which six are shown in Fig. 8. In each case, a generic LFAS type monostatic sonar was placed at $9.47^{\circ}$ longitude, $43.5^{\circ}$ latitude at a depth of $20 \mathrm{~m}$. The resulting standard deviation in signal excess and probability of detection are shown in Fig. 9.

Due to the complicated nature of underwater sound propagation, a small change in sound speed profile gradient in one location may result in a large change in signal excess at another location. It can be seen in Fig. 8 that the region to the east of the sensor position remains fairly constant-note that the samples shown in the figure were those with greatest deviation from the mean. Also the region at the extreme west of the scenario appears fairly constant-corresponding to deep water and more stable sound speed profile. An initial inspection shows that the uncertainty in signal excess appears to increase with sea bed slope. This may be seen in Fig. 9b in which standard deviation is plotted together with bathymetry contours.

\section{Discussion and conclusion}

This study has detailed a number of generic decision support techniques that may be applied to the problem of multistatic sonar network design. It is envisaged that such techniques will be wrapped around the existing MSTPA to provide a decision support functionality. The acoustic tool becomes the central metric calculator within a greater scheme of decision support concepts. Such a scheme will close the loop between rapid environmental assessment and the eventual operational performance of deployed assets. Such a tool may be used on board a platform in order to assist the tactical decisions of the operator. The tool therefore transitions from a planning aid which may be used prior to the deployment of assets (employing Monte Carlo simulations of potential scenarios), to a more operational tool on board the assets themselves providing real-time decision support.

The generic nature of the decision support techniques will allow for integration into different scenarios. This is the aim of the Environmental Knowledge and Operational Effectiveness program in which a number of scenarios, the performance of each being a function of environmental measurements, will be con- sidered for inclusion in decision support tools. These include:

- Multistatic network deployment (considered here)

- Optimal deployment of gliders for environmental sampling

- Planning naval interdiction against piracy

The transition of an otherwise scientific tool, used as a central metric calculator, into a more operational decision support system is a powerful ideal. This will facilitate the timely transition of scientific research tools into more operational scenarios.

The initial investigation of the effect of uncertainty within oceanographic predictions will form the basis for more detailed studies conducted during 2011. This study has demonstrated the resulting uncertainty in acoustic coverage as a function of uncertainty in extrapolated temperature and salinity predictions. An important result will be the sensitivity of the eventual optimum sensor locations to this uncertainty.

Open Access This article is distributed under the terms of the Creative Commons Attribution Noncommercial License which permits any noncommercial use, distribution, and reproduction in any medium, provided the original author(s) and source are credited.

\section{References}

Been R, Hughes D, Potter J, Strode C (2010) Cooperative anti submarine warfare at NURC: moving towards a net-centric capability. OCEANS

Burstein F, Holsapple CW (2008) Handbook on decision support systems 1 -basic themes. Springer

Chen CT, Millero FJ (1977) Speed of sound in sea water at high pressures. J Acoust Soc Am 62:1129-1135

Coraluppi S (2003) Multistatic sonar localization analysis. Tech. Rep. SR-377. NATO Undersea Research Centre, La Spezia

Davis PK, Kulick J, Egner M (2005) Implications of modern decision science for military decision-support systems. RAND, Santa Monica. http://www.loc.gov/catdir/toc/ecip0512/ 2005013419.html

Dechter R (1985) Generalized best-first search strategies and the optimality of A*. J Assoc Comput Mach 32(3):505536

Harrison CH (2002) Formulae for bistatic signal and reverberation. Tech. Rep. SR-371. SACLANTCEN, La Spezia

Hughes D, Been R, Kemna S (2010) Sensible behaviour strategies for AUVs in ASW scenarios. OCEANS, Sydney

Lenartz F, Mourre B, Barth A, Beckers JM, Vandenbulcke L, Rixen M (2010) Enhanced ocean temperature forecast skills through 3-D super-ensemble multi-model fusion. Geophys Res Lett 37(L19606). doi:10.129/2010GL044591

LePage KD (2010) An SNR maximization behaviour for autonomous AUV control. ECUA2010, Istanbul

Owen G (1995) Game theory, 3rd edn. Academic, New York

Rosenberg B (2010) Getting inside the enemy's decision cycle. Defense Systems 4(4):11-13 
Streichert F, Ulmer H (2005) JavaEvA — a java framework for evolutionary algorithms. Tech. rep. Centre for Bioinformatics Tubingen, University of Tubingen, Tubingen

Strode C (2009) Using the A* path planning algorithm to assess multistatic field performance. Tech. Rep. NURC Formal Report (FR series) NURC-FR-2009-010. NATO Undersea Research Centre, La Spezia

Von-neumann J, Morgenstern O (1953) Theory of games and economic behaviour, 3rd edn. Princeton University Press, Princeton

Wagner DH, Mylander WC, Sanders TJ (1999) Naval operations analysis, 3rd edn. Naval Institute, Annapolis

Wali Van Lohuizen C (1986) Knowledge management and policymaking. Sci Commun 8(1):12-38. doi:10.1177/ 107554708600800102
Wathelet R, Strode C, Vermeij A (2006) Advances in the Multistatic Tactical Planning Aid. Tech. Rep. NURC Formal Report (FR series) NURC-FR-2006-022. NATO Undersea Research Centre, La Spezia

Wathelet A, Strode C, Vermeij A, Been R (2008a) Optimisation in the Multistatic Tactical Planning Aid (MSTPA). Tech. Rep. NURC Formal Report (FR series) NURCFR-2008-013. NATO Undersea Research Centre, La Spezia

Wathelet R, Vermeij A, Strode C (2008b) Track classification model for the Multistatic Tactical Planning Aid. Tech. Rep. NURC Formal Report (FR series) NURC-FR-2008-006. NATO Undersea Research Centre, La Spezia

Williams JD (1954) The compleat strategist. McGraw-Hill, New York 